Opto;genetic stimulation of a hippocampal engram activates fear memory recall. Nature 484: 381-385.

Ramirez S, Liu X, MacDonald CJ, Moffa A, Zhou J, Redondo RL et al (2015). Activating positive memory engrams suppresses depression-like behaviour. Nature 522: 335-339.

Ryan TJ, Roy DS, Pignatelli M, Arons A, Tonegawa S (2015). Engram cells retain memory under retrograde amnesia. Science 348: 1007-1013.

Semon R (1904). Die mneme [English tranlsation: The mneme]. Wilhelm Engelmann: Leipzig.

Squire LR (1982). The neuropsychology of human memory. Annu Rev Neurosci. 5: 241-273.

Tonegawa S, Liu X, Ramirez S, Redondo RL (2015). Memory engram cells have come of age. Neuron 87: 918-931.

Neuropsychopharmacology Reviews (2016) 41, 370-371; doi: I0.1038/npp.20I5.264

\section{Extracellular Vesicles: Goodies for the Brain?}

Brain homeostasis requires extensive signaling and information exchange between all types of neural cells, including neurons and glia. Recent studies indicated a pivotal role of extracellular vesicles (EVs) in communication between neural cells and furthermore, in the conversation between neural cells and the periphery. EVs comprise a group of varied secreted vesicles (plasma membranederived microvesicles and endosomederived exosomes), which recently came into focus regarding their ability to shuttle biomolecules including RNA between cells and their potential to phenotypically modulate target cells. Apparently, all types of neural cells release EVs, which have been implicated in several physiological and pathological processes such as neuromodulation, synaptic plasticity, neuron-glia-interaction, and the spreading of neuropathological agents. Notably, EVs with the characteristics of exosomes seem to have a remarkable role in neuroprotection and neuroregeneration. Oligodendrocytes release exosomes in response to neurotransmitter signaling, that transfer cargo to neurons and enhance the tolerance of recipient neurons toward different types of cell stress (Frühbeis et al,
2013). These exosomes convey multilevel information by transferring stress-protective enzymes (Hsp70, SOD1, and catalase), activation of pro-survival signaling pathways and modulation of gene expression. In similar fashion, EVs secreted by Schwann-cells are internalized by neurons in the peripheral nervous system and promote axonal regeneration after injury by increasing axon elongation (Lopez-Verrilli et al, 2013). Thus, EVs transferred from myelinating glia cells to neurons convey neuroprotective and pro-regenerative messages and provide local support to facilitate axonal maintenance, homeostasis, and axonal growth. It is therefore conceivable that application of glial exosomes may offer a therapeutic opportunity to benefit neurons and prevent axonal death in the course of demyelinating diseases or other sorts of neural injury.

Moreover, there is compelling evidence that EVs released by cells in the periphery can enter the CNS and accomplish pro-neural activity. EVs derived from hematopoietic cells are able to pass the blood-brain barrier and deliver genetic information in form of mRNA and miRNAs to CNS neurons, in particular under inflammatory conditions (Ridder et al, 2014). It has been suggested that IFN $\gamma$ stimulated dendritic cells release EVs that promote CNS myelination and might be applied for remyelination therapies (Pusic et al, 2014). Furthermore, recent developments in the field of cell therapy strongly suggest that the systemic regenerative potential of stem cells observed in several neurological disorders is not revealed by cell engraftment, but largely due to paracrine signals delivered by EVs entering the CNS or modulating inflammatory responses. In a rat model of ischemia, intravenous administration of EVs originating from mesenchymal stromal cells improved functional recovery, which was related to enhanced neurite remodeling, neurogenesis, and neovascularization due to EV-mediated transfer of miRNAs to neural target cells (Xin et al, 2013). Neural stem cells (NSCs), which facilitate functional recovery upon systemic application in a number of neural diseases, release EVs after exposure to proinflammatory cytokines that are considered to mediate immunomodulation in the host environment (Cossetti et al, 2014).

In conclusion, EVs derived from cells within the nervous system as well as EVs entering the CNS from the periphery emerge as potent conveyors of complex messages in benefit of neural health. Future studies will be needed to uncover their full potential as therapeutic agents and to unravel their mode of action.

\section{FUNDING AND DISCLOSURE}

The authors declare no conflict of interest.

\section{ACKNOWLEDGMENTS}

Research of EMKA is supported by Deutsche Forschungsgemeinschaft grants KR3668/1-1 and KR3668/2-1. WPKE received a fellowship from the Focus Program Translational Neuroscience JGU Mainz.

\section{Eva-Maria Krämer-Albers ${ }^{1,2}$ and Wen Ping Kuo-Elsner ${ }^{1,2}$}

${ }^{1}$ Department of Molecular Cell Biology, Johannes Gutenberg University Mainz, Mainz, Germany; ${ }^{2}$ Focus Program Translational Neurosciences, Johannes Gutenberg University Mainz, Mainz, Germany

E-mail: emkraemer@uni-mainz.de

Cossetti C, Iraci N, Mercer TR, Leonardi T, Alpi E, Drago D et al (2014). Extracellular vesicles from neural stem cells transfer IFN-gamma via Ifngr1 to activate Stat1 signaling in target cells. $\mathrm{Mol}$ Cell 56: 193-204.

Frühbeis C, Fröhlich D, Kuo WP Amphornrat J, Thilemann S, Saab AS et al (2013). Neurotransmitter-triggered transfer of exosomes mediates oligodendrocyte-neuron communication. PLOS Biol 11: e1001604.

Lopez-Verrilli MA, Picou F, Court FA (2013). Schwann cell-derived exosomes enhance axonal regeneration in the peripheral nervous system. Glia 61: 1795-1806.

Pusic AD, Pusic KM, Clayton BL, Kraig RP (2014). IFNgamma-stimulated dendritic cell exosomes as a potential therapeutic for remyelination. J Neuroimmunol 266: 12-23.

Ridder K, Keller S, Dams M, Rupp AK, Schlaudraff J, Turco DD et al (2014). Extracellular vesiclemediated transfer of genetic information between the hematopoietic system and the brain in response to inflammation. PLOS Biol 12: e1001874.

Xin H, Li Y, Cui Y, Yang JJ, Zhang ZG, Chopp M (2013). Systemic administration of exosomes 
released from mesenchymal stromal cells promote functional recovery and neurovascular plasticity after stroke in rats. J Cereb Blood Flow Metab 33 1711-1715.

Neuropsychopharmacology Reviews (2016) 41, 37I-372; doi: I 0.1 038/npp.2015.242

\section{New Signaling Pathway for Gut-Brain Interactions}

Inflammatory Bowel Disease (IBD), which comprises Crohn's disease and ulcerative colitis, is a chronic inflammatory condition with a relapsing course. As IBD is associated with psychiatric disorders such as depression and anxiety as well as cognitive impairment, it was suggested that these psychological factors might predispose an individual to develop the disease. Now it is clear that there is bidirectional communication between the gut and the central nervous system (Kennedy et al, 2012) and our recent finding identifies a new mechanism by which IBD might cause behavioral manifestations (Zonis et al, 2015).

The generation of new neurons continues throughout adulthood in the subgranular zone of the dentate gyrus of the hippocampus. It is well accepted that adult hippocampal neurogenesis is involved in memory and learning (Aimone et al, 2014) and various aspects of emotion and the stress response (Cameron and Glover, 2015). Thus, disruption of hippocampal neurogenesis could have profound effects on a wide range of behaviors. Among many other factors, inflammation and pro-inflammatory cytokines negatively affect neurogenesis. Peripheral inflammation can signal the brain by activating the vagus nerve and Toll-like receptors in the circumventricular organs, and pro-inflammatory cytokines can enter the brain through saturable transport systems. Engagement of this immune-to-brain communication ultimately leads to the activation of resident microglia, which is a major source of pro-inflammatory cytokines in the brain.

Previously we found that during acute systemic inflammation, cytokines upregulated in the hippocampus trigger p21 ${ }^{\text {Cip1 }}$ (p21) induction in cells of neuronal lineage (Zonis et al, 2013). p21 is a cyclin-dependent kinase inhibitor that restrains cell cycle progression, thereby reducing neurogenesis. Neuronal progenitors treated in vitro with the pro-inflammatory cytokine interleukin-6 (IL-6) exhibit p21 induction and decreased proliferation, whereas IL-6 had no effect on the proliferation of progenitor cells derived from mice lacking p21. Thus, a direct inhibitory effect of IL-6 on neurogenesis is mediated by the induction of $\mathrm{p} 21$.

Unlike acute transient inflammation, chronic inflammatory disease might have continuing and long-lasting effects on neurogenesis. To assess the effects of chronic peripheral inflammation, we utilized the dextran sodium sulfate mouse model of IBD (Strober et al, 2002). This model produces colonic epithelial cell lesions and later chronic intestinal inflammation beginning 20 days after treatment. We found increased plasma levels of IL-6, indicative of the presence of systemic inflammation and this was accompanied by increased expression of Ibal, a marker of activated microglia, and the induct-

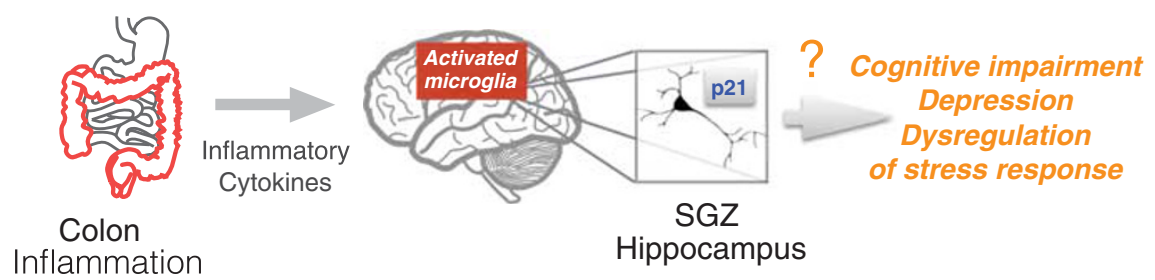

Figure 1. A proposed model for gut-hippocampus interaction. Peripheral inflammatory cytokines released during chronic intestinal inflammation activate microglia with subsequent induction of cytokines and p21 in early neuronal progenitors, effectively halting hippocampal neurogenesis and affecting behavior. SGZ-subgranular zone. ion of IL-6, IL-1 $\beta$, and p21 in the hippocampus. We also found a decrease in the number of newly developing neurons, likely due to cytokine-induced p21 expression in early neuronal progenitors. Subsequent in vitro experiments with neuronal progenitor cells confirmed that in addition to IL-6, the pro-inflammatory cytokines IL- $1 \beta$, and TNF- $\alpha$ also increase p21 expression (Zonis et al, 2015).

Our findings demonstrate that cytokine-induced p21 might have an important role in restraining neurogenesis during acute and chronic inflammation. These data reveal a previously unknown and potentially important signaling pathway for gut-brain interactions. Continuous immune signaling as a consequence of peripheral inflammation occurs in many chronic disorders, such as autoimmune disease, cancer, diabetes and obesity, and these illnesses manifest behavior abnormalities including cognitive impairment and depression. It is possible that the disruption of hippocampal neurogenesis might underlie some of the behavioral sequelae of IBD and other disorders associated with chronic inflammation (Figure 1).

\section{FUNDING AND DISCLOSURE}

This work was supported by NIH grant MH79988 and NARSAD Independent Investigator Award (VC). The authors declare no conflict of interest.

\section{ACKNOWLEDGMENTS}

The authors thank Dr Kolja Wawrowsky for help with the Figure 1.

\section{Vera Chesnokova ${ }^{1}$ and Robert N Pechnick ${ }^{2}$}

${ }^{1}$ Department of Medicine, Cedars-Sinai Medical Center, Los Angeles, CA, USA; ${ }^{2}$ Department of Basic Medical Sciences, College of Osteopathic Medicine of the Pacific, Western University of Health Sciences,

Pomona, CA, USA

E-mail: Vera.Chesnokova@cshs.org

Aimone JB, Li Y, Lee SW, Clemenson GD, Deng W, Gage FH (2014). Regulation and function of adult neurogenesis: from genes to cognition. Physiol Rev 94: 991-1026.

Cameron HA, Glover LR (2015). Adult neurogenesis: beyond learning and memory. Annu Rev Psychol 66: $53-81$. 